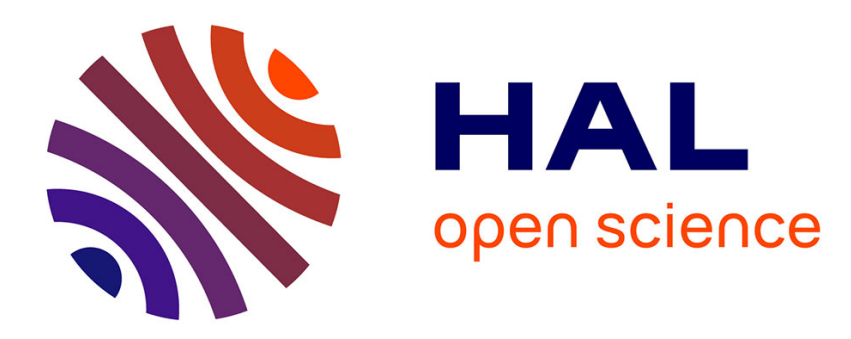

\title{
Coverings of singular curves over finite fields
}

\author{
Yves Aubry, Marc Perret
}

\section{To cite this version:}

Yves Aubry, Marc Perret. Coverings of singular curves over finite fields. Manuscripta mathematica, 1995, 88 (1), pp.467-478. 10.1007/BF02567835 . hal-00976489

\section{HAL Id: hal-00976489 \\ https://hal.science/hal-00976489}

Submitted on 11 Apr 2014

HAL is a multi-disciplinary open access archive for the deposit and dissemination of scientific research documents, whether they are published or not. The documents may come from teaching and research institutions in France or abroad, or from public or private research centers.
L'archive ouverte pluridisciplinaire HAL, est destinée au dépôt et à la diffusion de documents scientifiques de niveau recherche, publiés ou non, émanant des établissements d'enseignement et de recherche français ou étrangers, des laboratoires publics ou privés. 


\section{Coverings of Singular Curves over Finite Fields}

\section{Yves Aubry $^{1}$, Marc Perret ${ }^{2}$}

1 Département de Mathématiques, Université de Caen

Esplanade de la Paix - 14032 Caen Cedex - France.

${ }^{2}$ Unité de Mathématiques, École Normale Supérieure de Lyon 46, allée d'Italie - 69363 Lyon Cedex 7 - France.

Received April 10, 1995;

in revised form September 14, 1995

We prove that if $f: Y \longrightarrow X$ is a finite flat morphism between two reduced absolutely irreducible algebraic projective curves defined over the finite field $\mathbb{F}_{q}$, then

$$
\left|\sharp Y\left(\mathbb{F}_{q}\right)-\sharp X\left(\mathbb{F}_{q}\right)\right| \leq 2\left(\pi_{Y}-\pi_{X}\right) \sqrt{q},
$$

where $\pi_{C}$ is the arithmetic genus of a curve $C$. As application, we give some character sum estimation on singular curves. 
In this paper, the word curve stands for a reduced absolutely irreducible algebraic projective curve defined over the finite field $\mathbb{F}_{q}$ with $q$ elements. If $X$ is a smooth curve, it has been shown by Weil in [6] that the number of $\mathbb{F}_{q}$-rational points of $X$, denoted by $\sharp X\left(\mathbb{F}_{q}\right)$, is related to the geometric genus $g_{X}$ by :

$$
\left|\sharp X\left(\mathbb{F}_{q}\right)-(q+1)\right| \leq 2 g_{X} \sqrt{q}
$$

(with an improvement of Serre using the integral part, see [5]). In fact, Weil's statement, involving the zeta function of $X$, is more precise. It implies that if there is a finite morphism $f: Y \longrightarrow X$ between two smooth curves $X$ and $Y$ having (geometric) genus $g_{X}$ and $g_{Y}$ respectively, then (see for instance [2], proposition 6)

$$
\left|\sharp Y\left(\mathbb{F}_{q}\right)-\sharp X\left(\mathbb{F}_{q}\right)\right| \leq 2\left(g_{Y}-g_{X}\right) \sqrt{q} .
$$

When $X$ is the projective line, this is exactly Weil's bound for $Y$.

On the other hand, the authors proved in [1] that if $X$ is a singular curve, then Weil's inequality (1) holds if one replaces the geometric genus $g_{X}$ of $X$ by its arithmetic genus $\pi_{X}$. The aim of this paper is to give a generalization of both (2) and (1) for singular curves. Namely, if $f: Y \longrightarrow X$ is a finite flat morphism between two singular curves, then

$$
\left|\sharp Y\left(\mathbb{F}_{q}\right)-\sharp X\left(\mathbb{F}_{q}\right)\right| \leq 2\left(\pi_{Y}-\pi_{X}\right) \sqrt{q}
$$

holds.

We will prove (3) in the third section. The proof goes as follows. Inequality (2) can be applied to the finite morphism $\tilde{f}: \tilde{Y} \longrightarrow \tilde{X}$ induced by $f$ on the smooth models of $X$ and $Y$ respectively. Furthermore the number of $\mathbb{F}_{q}$-rational points of a curve is related to the number of $\mathbb{F}_{q}$-rational points of its smooth model (first section). Unfortunately, this is not sufficient to prove (3). One has to introduce (in the second section) the auxiliary curve $Z=\tilde{X} \times_{X} Y$, birational to $Y$. 
Finally, we apply this result in a fifth section to obtain some character sum estimations.

\section{A lemma}

The following lemma relates the number of $\mathbb{F}_{q}$-rational points of a curve and that of its smooth model. It is given in [1], but in order to be self contained, we give here its short proof. If $P$ is a $\mathbb{F}_{q}$-rational point of a curve $X$, we denote by $\alpha_{P}$ (respectively $\alpha_{P}(\infty)$ ) the number of $\mathbb{F}_{q}$-rational points (respectively of $\overline{\mathbb{F}}_{q}$-rational points, where $\overline{\mathbb{F}}_{q}$ stands for an algebraic closure of $\mathbb{F}_{q}$ ) of $\tilde{X}$, lying over $P$ in the normalization map $\nu_{X}: \tilde{X} \longrightarrow X$. Let $\mathcal{O}_{P}$ be the local ring of $X$ at $P$, and $\overline{\mathcal{O}_{P}}$ its integral closure in the function field $\mathbb{F}_{q}(X)$ of $X$. The quotient $\overline{\mathcal{O}_{P}} / \mathcal{O}_{P}$ is a $\mathbb{F}_{q}$-vector space of finite length : let $\delta_{P}$ be its dimension.

Lemma 1. Let $X$ be a reduced absolutely irreducible projective algebraic curve defined over $\mathbb{F}_{q}$. Then

$$
\left|\sharp \tilde{X}\left(\mathbb{F}_{q}\right)-\sharp X\left(\mathbb{F}_{q}\right)\right| \leq \sum_{P \in X\left(\mathbb{F}_{q}\right)}\left|\alpha_{P}-1\right| \leq \pi_{X}-g_{X} .
$$

Proof. Let us first prove that if $P$ is a $\mathbb{F}_{q}$-rational singular point of $X$, then $\alpha_{P}-1 \leq \delta_{P}$. Let $Q_{1}, \ldots, Q_{\alpha_{P}(\infty)}$ be the $\overline{\mathbb{F}}_{q}$-rational points of $\tilde{X}$ lying over $P$ (the $\alpha_{P}$ first beeing the $\mathbb{F}_{q}$-rational ones), and $\phi$ the $\mathbb{F}_{q}$-linear map

$$
\begin{array}{rlc}
\phi: \overline{\mathcal{O}_{P}} & \longrightarrow & \mathbb{F}_{q}^{\alpha_{P}} \\
f & \longmapsto\left(f\left(Q_{i}\right)\right)_{1 \leq i \leq \alpha_{P}}
\end{array}
$$

We prove that $\phi$ is onto : let $\left(x_{1}, \ldots, x_{\alpha_{P}}\right) \in \mathbb{F}_{q}^{\alpha_{P}}$ and $f_{i}=x_{i} \in \mathbb{F}_{q} \subset \mathbb{F}_{q}(X)$ if $i \leq \alpha_{P}$. For $i \geq \alpha_{P}+1$, let $f_{i}=0$. Then by the weak approximation theorem, there exists $g \in \mathbb{F}_{q}(X)$ such that $v_{Q_{i}}\left(g-f_{i}\right) \geq 1$ for $1 \leq i \leq \alpha_{P}(\infty)$. Hence, $\phi(g)=\left(x_{1}, \ldots, x_{\alpha_{P}}\right)$ and

$$
g \in \bigcap_{1 \leq i \leq \alpha_{P}(\infty)} \mathcal{O}_{Q_{i}}=\overline{\mathcal{O}_{P}}
$$


Since $f\left(Q_{1}\right)=\cdots=f\left(Q_{\alpha_{P}}\right)$ for $f \in \mathcal{O}_{P}$, it follows that $\phi\left(\mathcal{O}_{P}\right)$ is contained in the vector-line $L \subset \mathbb{F}_{q}{ }^{\alpha_{P}}$ spanned by $(1, \ldots, 1)$. One obtains a surjective linear map

$$
\tilde{\phi}: \overline{\mathcal{O}_{P}} / \mathcal{O}_{P} \longrightarrow \mathbb{F}_{q}{ }^{\alpha_{P}} / L .
$$

Taking dimensions, we obtain that $\alpha_{P}-1 \leq \delta_{P}$.

Now, Lemma 1 follows from the formulas

$$
\pi_{X}-g_{X}=\sum_{P \in \operatorname{Sing} X\left(\overline{\mathbb{F}}_{q}\right)} \delta_{P}
$$

and

$$
\sharp \tilde{X}\left(\mathbb{F}_{q}\right)-\sharp X\left(\mathbb{F}_{q}\right)=\sum_{P \in X\left(\mathbb{F}_{q}\right)}\left(\alpha_{P}-1\right) .
$$

\section{An auxiliary curve}

Let $X$ and $Y$ be two curves, and $f: Y \longrightarrow X$ be a finite flat morphism. In order to give an estimate for the difference between the numbers of $\mathbb{F}_{q}$-rational points of $X$ and $Y$, it is convenient to consider the fibre product $Z=\tilde{X} \times_{X} Y$.

Lemma 2. $Z$ is a reduced absolutely irreducible projective curve.

Proof. The map $f$ beeing finite, the map $Z \longrightarrow \tilde{X}$ is finite and onto, which implies that $\operatorname{dim} Z=\operatorname{dim} \tilde{X}=1$, and $Z$ is a curve.

In order to prove that $Z$ is absolutely integral, one has to prove that given an affine open set $\operatorname{Spec} A\left(X_{i}\right)$ of $X$, the ring $A=\overline{A\left(X_{i}\right)} \otimes_{A\left(X_{i}\right)} A\left(Y_{i}\right)$ is an integral domain, where $\bar{A}$ stands for the integral closure of a ring $A$, and $A\left(Y_{i}\right)$ is defined by $\operatorname{Spec} A\left(Y_{i}\right)=f^{-1}\left(\operatorname{Spec} A\left(X_{i}\right)\right)$. Denote by $\operatorname{Frac}(A)$ the quotient field of a domain $A$. By the flatness hypothesis,

$$
0 \longrightarrow \overline{A\left(X_{i}\right)} \longrightarrow \operatorname{Frac}\left(A\left(X_{i}\right)\right)
$$


induce

$$
0 \longrightarrow A \longrightarrow \operatorname{Frac}\left(A\left(X_{i}\right)\right) \otimes_{A\left(X_{i}\right)} A\left(Y_{i}\right)
$$

Then, the injective map

$$
\operatorname{Frac}\left(A\left(X_{i}\right)\right) \otimes_{A\left(X_{i}\right)} A\left(Y_{i}\right) \hookrightarrow \operatorname{Frac}\left(A\left(Y_{i}\right)\right)
$$

proves that $A$ is an integral domain, as a subring of a field.

Finally, $Z$ is projective. Indeed, $f$ is proper since it is finite, and $Z \longrightarrow \tilde{X}$ is also proper, so that the composite morphism $Z \longrightarrow \tilde{X} \longrightarrow \operatorname{Spec} \mathbb{F}_{q}$ is proper since $\tilde{X}$ is complete. Hence, $Z$ is projective as a complete curve.

By the universal properties of the fibre product and of normalization maps, one can write the following diagram, where all triangles and squares are commutative, and all morphisms are finite :

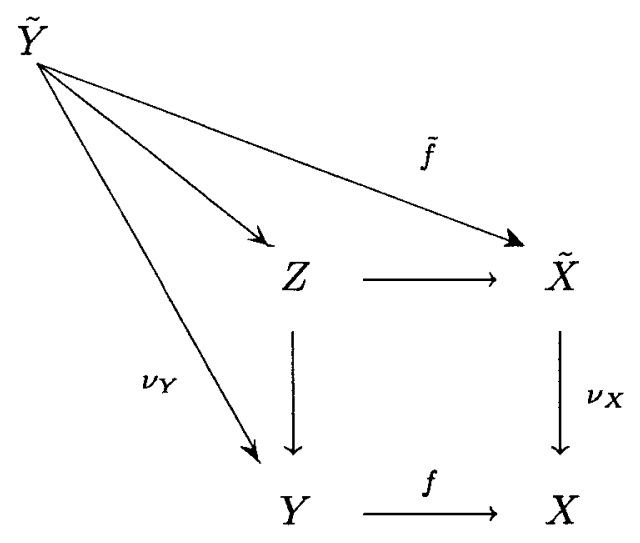

The sheaf of $\mathcal{O}_{X}$-modules $f_{*} \mathcal{O}_{Y}$ is coherent because $f$ is finite. It is then locally free since $f$ is flat. After localization, the stalks $\left(f_{*} \mathcal{O}_{Y}\right)_{P}$ are $\mathcal{O}_{X, P}$-module of finite type, whose rank doesn't depend on $P$ since $X$ is connected. Let 


$$
\begin{aligned}
r & =\operatorname{dim}_{\mathbb{F}_{q}(P)}\left(f_{*} \mathcal{O}_{Y}\right)_{P} \otimes_{\mathcal{O}_{X, P}} \mathbb{F}_{q}(P) \\
& =\sum_{Q \in f^{-1}(P)} \operatorname{dim}_{\mathbb{F}_{q}(P)}\left(\mathcal{O}_{Y, Q} \otimes_{\mathcal{O}_{X, P}} \mathbb{F}_{q}(P)\right)
\end{aligned}
$$

be this rank, where $\mathbb{F}_{q}(P)$ denotes the residue field of the point $P$. Note that if $r=1$, then $f^{-1}(P)=\{Q\}$ contains only one element for any $P \in X$, and $f$ induces a morphism of local rings $\mathcal{O}_{Y, Q} \longrightarrow \mathcal{O}_{X, P}$, so that $f$ is an isomorphism. Hence, one can suppose $r \geq 2$.

Proposition 3. (i) $Z$ is birational to $Y$. In particular, $g_{Z}=g_{Y}$.

(ii) The arithmetic genus of $Z$ is given by

$$
\pi_{Z}=\pi_{Y}-r\left(\pi_{X}-g_{X}\right) .
$$

Proof. (i) This is trivial since there are dominant morphisms $\tilde{Y} \longrightarrow Z$ and $Z \longrightarrow Y$.

(ii) Since both arithmetic and geometric genus of a curve $C$ and of $C \times_{\mathbb{F}_{q}} \overline{\mathbb{F}}_{q}=\bar{C}$ are the same, it is sufficient to compute $\pi_{\bar{Z}}$. To simplify notations, we continue to denote by $X, Y$ and $Z$ the curves $\bar{X}, \bar{Y}$ and $\bar{Z}$ respectively. The exact sequence of $\mathcal{O}_{X}$-module sheaves

$$
0 \longrightarrow \mathcal{O}_{X} \longrightarrow \nu_{X, *} \mathcal{O}_{\tilde{X}} \longrightarrow\left(\nu_{X, *} \mathcal{O}_{\tilde{X}}\right) / \mathcal{O}_{X} \longrightarrow 0
$$

tensorized by the flat $\mathcal{O}_{X}$-module $f_{*} \mathcal{O}_{Y}$, gives the exact sequence

$$
\begin{gathered}
0 \longrightarrow f_{*} \mathcal{O}_{Y} \longrightarrow\left(\nu_{X, *} \mathcal{O}_{\tilde{X}}\right) \otimes_{\mathcal{O}_{X}} f_{*} \mathcal{O}_{Y} \longrightarrow \\
\longrightarrow\left(\left(\nu_{X, *} \mathcal{O}_{\tilde{X}}\right) / \mathcal{O}_{X}\right) \otimes_{\mathcal{O}_{X}} f_{*} \mathcal{O}_{Y} \longrightarrow 0
\end{gathered}
$$

from which we deduce a long exact sequence of cohomology. Let us scrutinize its different terms. 
Since $H^{i}\left(X, f_{*} \mathcal{O}_{Y}\right) \cong H^{i}\left(Y, \mathcal{O}_{Y}\right)$ and $Y$ is projective, then we have $\operatorname{dim}_{\overline{\mathbb{F}}_{q}} H^{0}\left(X, f_{*} \mathcal{O}_{Y}\right)=1$ and $\operatorname{dim}_{\overline{\mathbb{F}}_{q}} H^{1}\left(X, f_{*} \mathcal{O}_{Y}\right)=\pi_{Y}$. In the same way,

$$
\operatorname{dim}_{\overline{\mathrm{F}}_{q}} H^{0}\left(X,\left(\nu_{X, *} \mathcal{O}_{\tilde{X}}\right) \otimes \mathcal{O}_{X} f_{*} \mathcal{O}_{Y}\right)=1
$$

and

$$
\operatorname{dim}_{\overline{\mathrm{F}}_{q}} H^{1}\left(X,\left(\nu_{X, *} \mathcal{O}_{\tilde{X}}\right) \otimes_{\mathcal{O}_{X}} f_{*} \mathcal{O}_{Y}\right)=\pi_{Z}
$$

Furthermore, the sheaf $\left(\nu_{X, *} \mathcal{O}_{\tilde{X}}\right) / \mathcal{O}_{X}$ is a sum of skyscraper sheaves on the singular points of $X$. Hence, this is also the case for $\left(\left(\nu_{X, *} \mathcal{O}_{\tilde{X}}\right) / \mathcal{O}_{X}\right) \otimes_{\mathcal{O}_{X}} f_{*} \mathcal{O}_{Y}$, and this prove the vanishing of its $H^{1}$. Finally,

$$
\begin{aligned}
& \operatorname{dim}_{\overline{\mathbb{F}}_{q}} H^{0}\left(X,\left(\left(\nu_{X, *} \mathcal{O}_{\tilde{X}}\right) / \mathcal{O}_{X}\right) \otimes_{\mathcal{O}_{X}} f_{*} \mathcal{O}_{Y}\right) \\
&=\sum_{P \in \operatorname{Sing} X\left(\overline{\mathbb{F}}_{q}\right)} \operatorname{dim}_{\overline{\mathbb{F}}_{q}}\left(\left(\overline{\mathcal{O}_{X, P}} / \mathcal{O}_{X, P}\right) \otimes_{\mathcal{O}_{X, P}}\left(f_{*} \mathcal{O}_{Y}\right)_{P}\right)= \\
& \quad \sum_{P \in \operatorname{Sing} X\left(\overline{\mathbb{F}}_{q}\right)} \operatorname{dim}_{\overline{\mathbb{F}}_{q}}\left(\left(\overline{\mathcal{O}_{X, P}} / \mathcal{O}_{X, P}\right) \otimes_{\overline{\mathbb{F}}_{q}}\left(\overline{\mathbb{F}}_{q} \otimes_{\mathcal{O}_{X, P}}\left(f_{*} \mathcal{O}_{Y}\right)_{P}\right)\right) \\
&\left.=\sum_{P \in \operatorname{Sing} X\left(\overline{\mathbb{F}}_{q}\right)} \operatorname{dim}_{\overline{\mathbb{F}}_{q}}\left(\overline{\left(\overline{\mathcal{O}}_{X, P}\right.} / \mathcal{O}_{X, P}\right) \otimes_{\overline{\mathbb{F}}_{q}}\left(\overline{\mathbb{F}}_{q}\right)^{r}\right) \\
&=\sum_{P \in \operatorname{Sing} X\left(\overline{\mathbb{F}}_{q}\right)} \operatorname{dim}_{\overline{\mathbb{F}}_{q}}\left(\overline{\mathcal{O}_{X, P}} / \mathcal{O}_{X, P}\right)^{r} \\
&= r\left(\pi_{X}-g_{X}\right) .
\end{aligned}
$$

The nullity of the alternating sum of the $\overline{\mathbb{F}}_{q}$-dimensions of the different terms of the long exact sequence of cohomology given by (4) gives :

$$
1-1+r\left(\pi_{X}-g_{X}\right)-\pi_{Y}+\pi_{Z}-0=0
$$

which was to be proved. 


\section{The main theorem}

Theorem 4. Let $X$ and $Y$ be two reduced absolutely irreducible projective algebraic curves defined over $\mathbb{F}_{q}$, with respective arithmetic genus $\pi_{X}$ and $\pi_{Y}$, and let $f: Y \longrightarrow X$ be a finite flat morphism defined over $\mathbb{F}_{q}$. Then

$$
\left|\sharp Y\left(\mathbb{F}_{q}\right)-\sharp X\left(\mathbb{F}_{q}\right)\right| \leq 2\left(\pi_{Y}-\pi_{X}\right) \sqrt{q} .
$$

The proof depends on the following lemma.

\section{Lemma 5.}

$\left|\left(\sharp Z\left(\mathbb{F}_{q}\right)-\sharp \tilde{X}\left(\mathbb{F}_{q}\right)\right)-\left(\sharp Y\left(\mathbb{F}_{q}\right)-\sharp X\left(\mathbb{F}_{q}\right)\right)\right| \leq(r-1)\left(\pi_{X}-g_{X}\right)$

(recall that $\left.r=\operatorname{dim}_{\mathbb{F}_{q}(P)}\left(f_{*} \mathcal{O}_{Y}\right)_{P} \otimes_{\mathcal{O}_{X, P}} \mathbb{F}_{q}(P)\right)$.

Proof. If $P \in X\left(\mathbb{F}_{q}\right)$, let

$$
\alpha_{P}=\sharp\left\{\tilde{P} \in \tilde{X}\left(\mathbb{F}_{q}\right) \mid \nu_{X}(\tilde{P})=P\right\}
$$

and

$$
\beta_{P}=\sharp\left\{Q \in Y\left(\mathbb{F}_{q}\right) \mid f(Q)=P\right\} .
$$

Then

$$
\beta_{P} \leq \sum_{Q \in f^{-1}(P)} 1 \leq \sum_{Q \in f^{-1}(P)} \operatorname{dim}_{\mathbb{F}_{q}(P)}\left(\mathcal{O}_{Y, Q} \otimes_{\mathcal{O}_{X, P}} \mathbb{F}_{q}(P)\right)=r
$$

Moreover,

$$
Z\left(\mathbb{F}_{q}\right)=\left\{(\tilde{P}, Q) \in \tilde{X}\left(\mathbb{F}_{q}\right) \times Y\left(\mathbb{F}_{q}\right) \mid \nu_{X}(\tilde{P})=f(Q)\right\}
$$

hence

$$
\begin{aligned}
\sharp Z\left(\mathbb{F}_{q}\right)-\sharp \tilde{X}\left(\mathbb{F}_{q}\right) & =\sum_{P \in X\left(\mathbb{F}_{q}\right)} \alpha_{P} \beta_{P}-\sum_{P \in X\left(\mathbb{F}_{q}\right)} \alpha_{P} \\
& =\sum_{P \in X\left(\mathbb{F}_{q}\right)} \alpha_{P}\left(\beta_{P}-1\right),
\end{aligned}
$$

and

$$
\begin{aligned}
\sharp Y\left(\mathbb{F}_{q}\right)-\sharp X\left(\mathbb{F}_{q}\right) & =\sum_{P \in X\left(\mathbb{F}_{q}\right)} \beta_{P}-\sum_{P \in X\left(\mathbb{F}_{q}\right)} 1 \\
& =\sum_{P \in X\left(\mathbb{F}_{q}\right)}\left(\beta_{P}-1\right) .
\end{aligned}
$$


By lemma 1, and since $r \geq 2$, we obtain

$$
\begin{aligned}
\mid\left(\sharp Z\left(\mathbb{F}_{q}\right)-\sharp \tilde{X}\left(\mathbb{F}_{q}\right)\right)- & \left(\sharp Y\left(\mathbb{F}_{q}\right)-\sharp X\left(\mathbb{F}_{q}\right)\right) \mid \leq \\
& \leq(r-1) \sum_{P \in X\left(\mathbb{F}_{q}\right)}\left|\alpha_{P}-1\right| \\
& \leq(r-1)\left(\pi_{X}-g_{X}\right) .
\end{aligned}
$$

The theorem follows easily from the preceding and the triangular inequality

$$
\begin{aligned}
\mid \sharp Y\left(\mathbb{F}_{q}\right)-\sharp X & \left(\mathbb{F}_{q}\right)|\leq| \sharp \tilde{Y}\left(\mathbb{F}_{q}\right)-\sharp Z\left(\mathbb{F}_{q}\right) \mid \\
& +\left|\left(\sharp Z\left(\mathbb{F}_{q}\right)-\sharp \tilde{X}\left(\mathbb{F}_{q}\right)\right)-\left(\sharp Y\left(\mathbb{F}_{q}\right)-\sharp X\left(\mathbb{F}_{q}\right)\right)\right| \\
& +\left|\sharp \tilde{X}\left(\mathbb{F}_{q}\right)-\sharp \tilde{Y}\left(\mathbb{F}_{q}\right)\right|
\end{aligned}
$$

\section{Remarks}

4.1. The proof of theorem 4 gives a better upper bound, namely

$$
\left|\sharp Y\left(\mathbb{F}_{q}\right)-\sharp X\left(\mathbb{F}_{q}\right)\right| \leq\left(\pi_{Y}-g_{Y}\right)-\left(\pi_{X}-g_{X}\right)+\left(g_{Y}-g_{X}\right)[2 \sqrt{q}] .
$$

See lemma 4.1 of [3] for the reason of the integer part $[2 \sqrt{q}]$ of $2 \sqrt{q}$.

4.2. It couldn't be expected theorem 4 to be true for any finite morphism $f: Y \longrightarrow X$. For instance, this is false for the normalization map of a singular curve. Here is another example : let $X$ be the plane curve $Y^{2} Z=X^{3}$. This is a singular cubic curve, its arithmetic genus is $\pi_{X}=1$, and for any integer $n$, we have $\sharp X\left(\mathbb{F}_{2^{n}}\right)=2^{n}+1$. Let us consider the morphism

$$
\pi: \begin{array}{ccc}
\mathbb{P}^{1} & \longrightarrow & X \\
(u: v) & \longmapsto & \left(u^{2} v: u^{3}: v^{3}\right)
\end{array}
$$

Suppose theorem 4 would be true for any finite morphism $f$, and let $C$ be any smooth curve of genus $g_{C}$, defined over $\mathbb{F}_{2}$. 
There is a finite morphism $\pi^{\prime}: C \longrightarrow \mathbb{P}^{1}$, hence by composition a finite morphism $f=\pi \circ \pi^{\prime}: C \longrightarrow X$, and theorem 4 would imply that for $q=2^{n}$,

$$
\left|\sharp C\left(\mathbb{F}_{q}\right)-(q+1)\right| \leq 2\left(g_{C}-1\right) \sqrt{q},
$$

which is false : one can consider for instance the smooth curve $C$ given by $X^{3}+Y^{3}+Z^{3}=0$, which has 9 rational points over $\mathbb{F}_{4}$ and genus $g_{C}=1$.

4.3. If $X$ is smooth (in particular if $X$ is the projective line), then any finite morphism $Y \longrightarrow X$ is flat. Indeed, if $f(Q)=P$, then the local ring $\mathcal{O}_{Y, Q}$ is a finitely generated $\mathcal{O}_{X, P}$-module without torsion element. But $\mathcal{O}_{X, P}$ is a principal ideal domain since $X$ is supposed to be smooth, so that $\mathcal{O}_{Y, Q}$ is a flat $\mathcal{O}_{X, P^{-}}$ module. This shows that theorem 4 contains all known results (1) and (2) of the introduction.

Note that there are many other situations where $Y \longrightarrow X$ is flat. This is the case for instance if $X=Y / G$, where $G$ is a finite group of automorphisms of $Y$ (see [4]), or (by stability of flatness by base change) if $f$ is the reduction modulo a prime ideal of a flat morphism between two curves defined over a number field, or (also by base change, see section 5 below) if $f$ is a Kummer, or an Artin-Schreier morphism.

\section{Application to exponential sums}

Let $X$ be a curve over a finite field $\mathbb{F}_{q}$ of odd characteristic and $f \in \mathbb{F}_{q}(X)$ a function on $X$. When $X$ is smooth, there is an extensive literature on estimations of the character sums $\sum_{P \in X\left(\mathbb{F}_{q}\right)}\left(\frac{f(P)}{q}\right)$, where $(\dot{\bar{q}})$ is the Legendre symbol on $\mathbb{F}_{q}$ (with the convention that $\left(\frac{f(P)}{q}\right)=0$ if $P$ is a zero or a pole of $f)$. In fact, this character sum equals to $\sharp Y\left(\mathbb{F}_{q}\right)-\sharp X\left(\mathbb{F}_{q}\right)$, where $Y$ is the Zariski closure of the curve $\left\{(P, y) \in X \times \mathbb{P}^{1} \mid\right.$ $\left.y^{2}=f(P)\right\}$ in the surface $X \times \mathbb{P}^{1}$. Indeed, there are exactly $1+\left(\frac{f(P)}{q}\right)$ points in $Y\left(\mathbb{F}_{q}\right)$ above a given point $P \in X\left(\mathbb{F}_{q}\right)$. 
The geometric genus $g_{Y}$ of $Y$ is given from $g_{X}$ by Hurwitz formula, and its arithmetic genus is given by the following lemma, whose proof is a standard and tedious calculation using $\check{C}$ ech cohomology.

Lemma 6. Let $Y$ be the Zariski closure of $\left\{(P, y) \in X \times \mathbb{P}^{1} \mid\right.$ $\left.y^{n}=f(P)\right\}$. Then, $\pi_{Y}=n \pi_{X}+(n-1)\left(\operatorname{deg}(f)_{0}-1\right)$, where $(f)_{0}$ denotes the divisors of zeroes of $f$.

Since the squaring map $\mathbb{P}^{1} \longrightarrow \mathbb{P}^{1}, y \longmapsto y^{2}$ is flat, then by base change $Y=X \times_{\mathbb{P}^{1}} \mathbb{P}^{1} \longrightarrow X$ is also flat. Hence, theorem 4 applies, so that the following holds :

\section{Theorem 7.}

$$
\left|\sum_{P \in X\left(\mathbb{F}_{q}\right)}\left(\frac{f(P)}{q}\right)\right| \leq 2(n-1)\left(\pi_{X}+\operatorname{deg}(f)_{0}-1\right) \sqrt{q}
$$

Of course, one can give a better upper bound using remark 4.1., and one can study additive character sums via ArtinSchreier coverings.

\section{References}

[1] Aubry Y., Perret M. : A Weil theorem for singular curves, Proceedings of Arithmetic, Geometry and Coding Theory IV, ed. Pellikaan, Perret, Vlăduţ. De Gruyter, (1995)

[2] Lachaud G. : Sommes d'Eisenstein et nombre de points de certaines courbes algébriques sur les corps finis, C. R. Acad. Sci. Paris 305, 729-732 (1987)

[3] Lachaud G. : Artin-Schreier curves, exponential sums and the CarlitzUchiyama bound for geometric codes, J. Number Theory 39, 18-40 (1991)

[4] Mumford D. : Abelian varieties, Oxford Univ. Press, Oxford 1970 
[5] Serre J.-P. : Sur le nombre de points rationnels d'une courbe algébrique sur un corps fini, C. R. Acad. Sci. Paris 296, série I, 397-402 (1983)

[6] Weil A. : Sur les courbes algébriques et les variétés qui s'en déduisent, Hermann, Paris 1948 